\title{
Subjective State, Blood Pressure, and Behavioral Control Changes Produced by an "Energy Shot"
}

\author{
Cecile A. Marczinski, PhD, ${ }^{1}$ Amy L. Stamates, BS, ${ }^{1}$ Julianne Ossege, PhD, ${ }^{2}$ \\ Sarah F. Maloney, BS, ${ }^{1}$ Mark E. Bardgett, $\mathrm{PhD},{ }^{1}$ and Clifford J. Brown, $\mathrm{BS}^{1}$
}

Background: Energy drinks and energy shots are popular consumer beverages that are advertised to increase feelings of alertness. Typically, these products include high levels of caffeine, a mild psychostimulant drug. The scientific evidence demonstrating the specific benefits of energy products to users in terms of subjective state and objective performance is surprisingly lacking. Moreover, there are rising health concerns associated with the use of these products. Therefore, the purpose of this study was to investigate the acute effects of a popular energy shot (5-Hour Energy ${ }^{\circledR}$ ) on subjective and objective measures that were assessed hourly for 6 hours following consumption.

Methods: Participants $(n=14)$ completed a three-session study where they received the energy shot, a placebo control, and no drink. Following dose administration, participants completed subjective Profile of Mood States ratings hourly for 6 hours. Participants also repeatedly completed a behavioral control task (the cued go/no-go task) and provided blood pressure and pulse rate readings at each hour.

Results: Consumption of the energy shot did improve subjective state, as measured by increased ratings of vigor and decreased ratings of fatigue. However, the energy shot did not alter objective performance, which worsened over time. Importantly, the energy shot elevated both systolic and diastolic blood pressure.

Conclusions: Consumption of one energy shot may only result in modest benefits to subjective state. Individuals with preexisting hypertension or other medical conditions should be cautious about using these new consumer products.

\section{Introduction}

$\mathbf{E}$ NERGY DRINKS AND ENERGY SHOTS are popular consumer products advertised to decrease lethargy and increase energy levels. Energy products differ in volume and typical ingredients, but most include high levels of caffeine, sugar, vitamins, and other stimulant ingredients. ${ }^{1}$ While product labeling for this category has improved substantially over the last few years, many energy product ingredients are still considered proprietary blends. Thus, products can differ considerably in the psychoactive compounds contained within them, including caffeine, and the stimulant ingredients are often listed on the product label under an imprecise category label called "Energy Blend."2 Caffeine is the most widely consumed psychoactive drug. ${ }^{3}$ The caffeine doses in energy drinks and energy shots are within the range observed in other caffeinated beverages, often similar to coffee but usually more caffeine than the majority of soft drinks. Rising concerns about the safety of energy products, even though caffeine is thought to be relatively safe, suggest that systematic investigation of the risks and benefits of product use is warranted. $^{4,5}$
Energy products are advertised to decrease fatigue, and it is likely that these caffeinated beverages are effective in that regard. The administration of moderate $(1-4 \mathrm{mg} / \mathrm{kg})$ doses of caffeine reliably produces mild stimulant effects, such as enhancing subjective ratings of stimulation and wakefulness while decreasing ratings of mental fatigue. ${ }^{1,6,7}$ Caffeine is more effective in enhancing feelings of stimulation in individuals who are fatigued. ${ }^{7,8}$ Energy products are also advertised to improve mental functioning, which is also partially supported by the literature. Moderate doses of caffeine and energy drinks can improve some, but not all, aspects of cognitive performance. Caffeine and caffeine-containing energy drinks have been shown to be able to improve attention, speed reaction times, improve memory, facilitate vigilance, and improve verbal reasoning. ${ }^{6,8-11}$ However, the benefits of caffeine appear more reliable for improving response speed and do not extend to other aspects of behavioral control, including the ability to inhibit (i.e., suppress) responses. ${ }^{1,12}$ Thus, caffeine is likely not a pure cognitive enhancer but instead enhances cognition via its action on general arousal levels such as mitigating fatigue. ${ }^{11}$ Finally, energy products are advertised as safe. However, the health risks that have been

Departments of ${ }^{1}$ Psychological Science and ${ }^{2}$ Advanced Nursing Studies, Northern Kentucky University, Highland Heights, Kentucky. 
associated with energy drinks have often been related to cardiovascular functioning. The acute effects of caffeine can moderately increase blood pressure and heart rate. ${ }^{13-15}$ While the elevation in blood pressure due to caffeine may be relatively innocuous in healthy individuals, it may be problematic in individuals who have high blood pressure. Moreover, the duration of elevations in blood pressure following energy product use remains unclear particularly in individuals who use these products repeatedly.

Given that the risks and benefits of energy shot use remain poorly elucidated in the literature, the present study examined the acute effects of an energy shot ( $2 \mathrm{oz} 5$-Hour Energy $\left.{ }^{\circledR}\right)$, compared to a placebo control and no drink condition using a within-subjects design. Only healthy young adults who had normal blood pressure participated. Following dose administration, participants completed hourly subjective ratings (Profile of Mood States) for 6 hours and repeatedly completed a behavioral control task (the cued go/no-go task). Repeated testing on this behavioral control task was predicted to induce fatigue in all participants. Blood pressure and pulse rate readings were recorded hourly. It was predicted that the energy shot should improve subjective state such as allaying perceived fatigue. For the behavioral control task, it was predicted that the energy shot might improve reaction times but would likely not impact response inhibition accuracy. Finally, it was predicted that the energy shot would moderately elevate blood pressure.

\section{Method}

\section{Participants}

Fourteen healthy individuals ( 7 males) between the ages of 18 and 29 years were recruited to participate in a three-session study where they received one of three conditions: 2 oz 5-Hour Energy ${ }^{\circledR}$ shot, 2 oz Ocean Spray ${ }^{\circledR}$ Diet Cranberry Juice as the placebo, or no drink. Ethnicity was self-reported as Caucasian $(n=7)$ or African American $(n=7)$. Potential volunteers completed questionnaires that provided demographic information and physical and mental health status. Any individual who self-reported a psychiatric disorder, substance abuse disorder, or head injury was excluded. All participants were tested for recent use of amphetamines, barbiturates, benzodiazepines, cocaine, ecstasy, methamphetamine, opiates, and tetrahydrocannabinol by urinalysis at the start of each session (uVera Diagnostics, Inc., Norfolk, VA). If any individual had tested positive for any of these drugs, they would have been excluded from the study. No females who were pregnant or breast-feeding participated, as determined by both self-report and urine human chorionic gonadotropin (HCG) levels. Eligibility requirements included being at least 18 years of age, having a body mass index $(\mathrm{BMI})$ in the normal range $(<25)$, having consumed at least one energy drink in the past year, and having consumed at least one caffeinated beverage in the past 2 weeks. The majority of participants $(64 \%)$ had tried 5-Hour Energy ${ }^{\circledR}$ at least once. All participants had normal color vision and normal or corrected-to-normal 20/20 vision. Demographic characteristics and typical caffeine use and typical use of 5-Hour Energy ${ }^{\circledR}$ are presented in Table 1. The Institutional Review Board at Northern Kentucky University approved this study. All volunteers provided informed consent prior to participating. Upon completion of the entire study, participants were compensated with $\$ 100$.
TABle 1. Demographic Characteristics and Typical Caffeine Use for All Participants $(N=14$ OF EQUAL GENDER $)$

\begin{tabular}{|c|c|c|}
\hline Variable & Mean & $\mathrm{SD}$ \\
\hline Age (years) & 20.07 & 2.84 \\
\hline Body weight (kg) & 71.16 & 13.11 \\
\hline Body mass index & 24.96 & 5.93 \\
\hline Self-reported daily caffeine use $(\mathrm{mg} / \mathrm{kg})^{\mathrm{a}}$ & 3.65 & 2.37 \\
\hline Weekly use of 5-Hour Energy ${ }^{\circledR}$ & 0.39 & 0.38 \\
\hline
\end{tabular}

${ }^{\text {a }}$ Participants were asked to report their typical daily caffeine use from all sources (coffee, tea, soft drinks, energy products, chocolate, and medications). Body weight was assessed on the first session. The average participant in this study reported daily caffeine consumption equivalent to a $12 \mathrm{oz}$ cup of drip coffee and similar to the caffeine reportedly found in one energy shot used in this study.

$\mathrm{SD}$, standard deviation.

\section{Materials}

Profile of Mood States (POMS) brief form. ${ }^{16,17}$. This 30adjective rating scale is used to assess current mood. Each adjective is rated on a 5-point scale that ranges from $0=$ "not at all" to $4=$ "extremely." Six mood scales are summed separately, which include Tension-Anxiety, Depression-Dejection, Anger-Hostility, Vigor-Activity, Fatigue-Inertia, and Confusion-Bewilderment. This scale is known to be sensitive to the acute administration of caffeine. ${ }^{8}$

Cued go/no-go task. Behavioral control was measured by the cued go/no-go task, a neurocognitive task that is known to be sensitive to the performance-enhancing effects of caffeine and energy drinks. ${ }^{1,12}$ For this task, participants are instructed to sit in front of a laptop computer and use the keyboard to make quick responses to go targets and to suppress responses to no-go targets. The go and no-go targets are rectangles that are green and blue respectively. For go targets, participants are instructed to press the forward slash (/) key as quickly as they can when a green rectangle appears. For no-go targets, participants are instructed not to make any action when a blue rectangle appears. A trial involves the following sequence of events: (1) a fixation point $(+)$ for $800 \mathrm{~ms}$; (2) a blank screen for $500 \mathrm{~ms}$; (3) a cue, displayed for one of five stimulus onset asynchronies (SOAs = 100, 200, 300, 400, and $500 \mathrm{~ms}$ ); (4) a target (go or no-go) that remains visible until a response is made or $1,000 \mathrm{~ms}$ elapses; and (5) an intertrial interval of $700 \mathrm{~ms}$. The cue is an empty rectangle displayed horizontally (height $=2.5$ $\mathrm{cm}$ and width $=7.5 \mathrm{~cm}$ ) or vertically (height $=7.5 \mathrm{~cm}$ and width $=2.5 \mathrm{~cm}$ ). The green go and blue no-go targets are displayed as solid color that fills the interior of the rectangle cue.

The cues in the task do not require a response, but they do signal the probability of the type of target that will likely follow. Cues presented vertically are followed by go targets on $80 \%$ of the trials and are followed by no-go targets on $20 \%$ of the trials. Cues presented horizontally are followed by no-go targets on $80 \%$ of the trials and are followed by go targets on $20 \%$ of the trials. Thus, vertical and horizontal cues function as go and no-go cues respectively. Therefore, trials where the cue incorrectly predicts the target are of most interest, given that this is where response prepotency is present and response conflict is maximal. The different SOAs between cues and targets ensure that participants cannot anticipate the exact onset of the targets. 
A 250-trial version of the cued go/no-go task was used in this study. An equal number of vertical (125) and horizontal (125) cues were presented before an equal number of go (125) and no-go (125) targets. Each cue-target combination was presented at each of the five SOAs, with presentation of cue-target combinations and SOAs occurring randomly. For each trial, the computer recorded whether a response occurred. If a response occurred, the reaction time (RT) in milliseconds was recorded. During the intertrial interval, "correct" or "incorrect" response feedback with the RT was provided to the participant. The test took about 12 minutes to complete. A Dell Latitude laptop using E-Prime 2.0 experiment generation software was used for this task. ${ }^{18}$

\section{Procedure}

Individuals were recruited for this study using posters that were displayed around the university. When potential volunteers contacted the lab by phone, they were informed that the purpose of the experiment was to examine the acute effects of caffeinated beverages, including energy drinks, on behavioral and mental functioning. They were informed that they would participate in three separate 7 hour sessions where they would be asked to perform computerized tasks, complete questionnaires, supply blood pressure readings, and provide a urine sample in a private bathroom. They were also informed that they may or may not receive a beverage to consume, and that beverage could contain caffeine. The maximum caffeine dose that could be administered was that found in a cup of coffee or two cans of a typical soft drink.

After the telephone screening, eligible volunteers were scheduled for three separate sessions (separated by a minimum of 24 hours and session start times beginning at approximately 10 a.m.). Session start times did not deviate by more than 1 hour for each individual subject. Dose administration for this study was double-blind, and dose order was counterbalanced between subjects. Before each session, participants were required to fast for 2 hours, abstain from caffeine for 8 hours, and abstain from alcohol for 24 hours. Any subject that failed to follow these instructions, as measured by their responses to a dietary questionnaire at the start of each session, was rescheduled. This only occurred on twice for the entire study, and the participant was rescheduled for testing the next day.

Upon arrival in the laboratory, participants provided informed consent and were assigned to the dose order for the three-session study, which was counterbalanced between subjects. The three dose conditions included a $2 \mathrm{oz} 5$-Hour Energy $^{\circledR}$ shot, 2 oz. Ocean Spray ${ }^{\circledR}$ Diet Cranberry Juice as the placebo, or no drink. The energy shot chosen (in berry flavor) is the market leader in the category and is sold in vending machines on the campus. The product label lists an energy blend of $1,870 \mathrm{mg}$, which includes unknown quantities of taurine, glucuronic acid, malic acid, N-acetyl L-tyrosine, Lphenylalanine, caffeine, and citicoline. The manufacturer's Web site reports that each bottle contains $200 \mathrm{mg}$ of caffeine (www.5hourenergy.com), an amount of caffeine that is similar to a strong cup of coffee. The placebo was chosen based on similarity in taste and color to the energy shot. Both the energy shot and placebo are sugar free. The no drink control condition was included to control for expectancy effects.

Participants were tested individually by a research assistant. All testing was conducted in a small room that consisted of a chair and desk with a laptop computer that operated the cued go/no-go task. On the first session only, participants completed a medical history form, the caffeine use questionnaire, and were weighed. They also completed one practice version of the cued go/no-go task so that they would be familiar with the task. Before dose administration on all three sessions, participants completed a baseline POMS and completed the cued go/no-go task. Blood pressure and pulse rate were also recorded using a Medline Automatic Blood Pressure Monitor (Medline Industries, Inc., Mundelein, IL) to ensure that normal blood pressure (i.e., below $120 / 80 \mathrm{~mm} \mathrm{Hg}$ ) was recorded. For readings, participants were asked to remove any bulky material from the left arm and rest the arm on the table with the palm facing up.

Participants then received their beverage (if assigned) in a small plastic cup and were informed that the drink should be consumed in less than a minute. Exact contents of the beverages were never disclosed to participants. Participants rested for 30 minutes before the onset of testing. Starting at 30 minutes following dose administration, the testing battery began. Participants were asked to complete the cued go/no-go task at 30, 90, 150, 210, 270, and 330 minutes after dose administration. Participants completed the POMS subjective ratings at 40, 100, 160, 220, 280, and 340 minutes after dose administration. Blood pressure and pulse rate were recorded at $60,120,180,240,300$, and 360 minutes after dose administration. When participants were not completing the task, questionnaires, or giving blood pressure readings, they were offered magazines to read during periods of rest. Upon completion of the testing period, participants were released. Upon completion of the third session, participants were debriefed and released. They received their compensation by check in the mail.

\section{Criterion measures and data analyses}

For the cued go/no-go task, there were two primary measures of interest: the speed of response to go targets and failure to inhibit responses to no-go targets. Speed of response to go targets was measured by the mean RT to go targets when preceded by a no-go cue for each test. Failure to inhibit responses (p-inhibition failures) were measured as the proportion of nogo target trials in which a participant failed to suppress a response when preceded by a go cue for each test. Both mean RTs and p-inhibition failures were analyzed by a $3 \times 6$ (Dose: energy shot, placebo, no drink $\times$ Time: $30,90,150,210$, 270,330 minutes) within-subjects analysis of variance (ANOVA). The POMS subjective ratings, blood pressure (systolic and diastolic), and pulse rate recordings were also submitted to a $3 \times 6$ (Dose $\times$ Time) within-subjects ANOVA. When overall ANOVAs required post-hoc testing, the least significant difference (LSD) test was utilized. All statistical analyses were performed using SPSS v18.0 (IBM Corp., Armonk, NY) with level of significance set at 0.05 for all statistical tests.

\section{Results}

\section{Subjective POMS ratings}

The mean POMS ratings for the six mood scales were not significantly different at baseline $(p>0.24)$. Mean (standard deviation $[S D]$ ) POMS ratings following dose administration are presented in Table 2. Results of the ANOVA for the POMS Vigor-Activity ratings revealed a significant main 
Table 2. Mean (SD) Profile of Mood States (POMS) Ratings for EAch Time Point for the Energy Shot, Placebo, and No Drink Conditions

\begin{tabular}{|c|c|c|c|c|c|c|}
\hline \multirow[b]{3}{*}{ Variable } & \multicolumn{6}{|c|}{ Dose condition } \\
\hline & \multicolumn{2}{|c|}{ No drink } & \multicolumn{2}{|c|}{ Placebo } & \multicolumn{2}{|c|}{ Energy shot } \\
\hline & $M$ & SD & $M$ & SD & $M$ & SD \\
\hline \multicolumn{7}{|c|}{ Vigor-Activity } \\
\hline 40 minutes & 2.93 & 3.10 & 5.43 & 3.80 & 6.50 & 3.80 \\
\hline 100 minutes & 2.50 & 3.01 & 3.71 & 2.73 & 5.14 & 3.66 \\
\hline 160 minutes & 2.21 & 2.75 & 3.43 & 3.13 & 6.07 & 5.28 \\
\hline 220 minutes & 1.64 & 2.82 & 2.43 & 2.41 & 4.93 & 4.86 \\
\hline 280 minutes & 1.86 & 2.69 & 2.43 & 2.90 & 4.50 & 5.32 \\
\hline 340 minutes & 1.86 & 2.25 & 2.14 & 2.88 & 3.93 & 4.68 \\
\hline \multicolumn{7}{|c|}{ Fatigue-Inertia } \\
\hline 40 minutes & 2.43 & 2.71 & 1.57 & 2.74 & 0.64 & 1.45 \\
\hline 100 minutes & 3.36 & 4.05 & 2.36 & 2.13 & .79 & 0.98 \\
\hline 160 minutes & 3.64 & 3.95 & 2.79 & 3.26 & 1.00 & 1.30 \\
\hline 220 minutes & 3.93 & 3.77 & 2.86 & 3.06 & 1.36 & 2.24 \\
\hline 280 minutes & 3.93 & 4.50 & 3.71 & 3.50 & 1.50 & 1.95 \\
\hline 340 minutes & 3.57 & 4.05 & 3.86 & 3.78 & 2.50 & 2.59 \\
\hline \multicolumn{7}{|c|}{ Confusion-Bewilderment } \\
\hline 40 minutes & 0.86 & 0.86 & 1.64 & 1.82 & 1.86 & 1.17 \\
\hline 100 minutes & 0.86 & 1.23 & 1.29 & 1.20 & 1.79 & 1.53 \\
\hline 160 minutes & 1.07 & 1.14 & 1.57 & 1.40 & 1.71 & 1.54 \\
\hline 220 minutes & 0.86 & 1.10 & 1.21 & 1.25 & 1.57 & 1.51 \\
\hline 280 minutes & 0.86 & 1.10 & 1.00 & 1.11 & 1.36 & 1.34 \\
\hline 340 minutes & 0.93 & 1.21 & 0.79 & 1.12 & 1.07 & 1.21 \\
\hline \multicolumn{7}{|c|}{ Tension-Anxiety } \\
\hline 40 minutes & 0.36 & 0.50 & 0.50 & 0.76 & 1.14 & 1.61 \\
\hline 100 minutes & 0.50 & 1.09 & 0.64 & 0.8 & 0.93 & 1.27 \\
\hline 160 minutes & 0.71 & 1.38 & 0.57 & 0.94 & 1.07 & 1.33 \\
\hline 220 minutes & 0.93 & 1.64 & 0.86 & 1.35 & 1.07 & 1.21 \\
\hline 280 minutes & 1.00 & 1.66 & 0.71 & 0.99 & 0.64 & 1.22 \\
\hline 340 minutes & 1.07 & 2.13 & 0.64 & 1.28 & 0.71 & 1.44 \\
\hline \multicolumn{7}{|c|}{ Depression-Dejection } \\
\hline 40 minutes & 0.36 & 1.34 & 0.00 & 0.00 & 0.29 & 0.47 \\
\hline 100 minutes & 0.21 & 0.80 & 0.00 & 0.00 & 0.21 & 0.43 \\
\hline 160 minutes & 0.36 & 1.34 & 0.07 & 0.27 & 0.21 & 0.43 \\
\hline 220 minutes & 0.50 & 1.61 & 0.14 & 0.36 & 0.21 & 0.43 \\
\hline 280 minutes & 0.50 & 1.87 & 0.14 & 0.36 & 0.00 & 0.00 \\
\hline 340 minutes & 0.57 & 2.14 & 0.14 & 0.54 & 0.00 & 0.00 \\
\hline \multicolumn{7}{|c|}{ Anger-Hostility } \\
\hline 40 minutes & 0.14 & 0.36 & 0.07 & 0.27 & 0.00 & 0.00 \\
\hline 100 minutes & 0.21 & 0.43 & 0.14 & 0.54 & 0.00 & 0.00 \\
\hline 160 minutes & 0.36 & 0.75 & 0.07 & 0.27 & 0.00 & 0.00 \\
\hline 220 minutes & 0.36 & 0.63 & 0.07 & 0.27 & 0.21 & 0.80 \\
\hline 280 minutes & 0.43 & 0.94 & 0.21 & 0.58 & 0.21 & 0.80 \\
\hline 340 minutes & 0.64 & 1.15 & 0.14 & 0.54 & 0.36 & 1.08 \\
\hline
\end{tabular}

$M$, mean; $S D$, standard deviation.

effect of Dose, $F(2,26)=8.38, p=0.002$, and a significant main effect of Time, $F(5,65)=4.36, p=0.002$. There was no significant interaction in this analysis $(p=0.60)$. Posthoc LSD tests for the main effect of Dose revealed that Vigor-Activity ratings were significantly higher following consumption of the energy shot when compared to both the placebo and no drink conditions $(p<0.043)$. The VigorActivity ratings did not differ for the placebo and no drink conditions $(p=0.10)$. The post-hoc LSD tests for the main effect of Time revealed that Vigor-Activity ratings declined with time. The Vigor-Activity ratings were significantly higher at the 40 minute time point when compared to all time points $(p<0.043)$, except that the 40 minute time point did not differ from the 160 minute time point ( $p=0.27)$.

Results of the ANOVA for the POMS Fatigue-Inertia ratings revealed a significant main effect of Dose, $F(2$, $26)=4.56, p=0.020$, and a significant main effect of Time, $F(5,65)=3.35, p=0.009$. There was no significant interaction in this analysis $(p=0.79)$. Post-hoc LSD tests for the main effect of Dose revealed that Fatigue-Inertia ratings were significantly lower following consumption of the energy shot when compared to both the placebo and no drink conditions $(p<0.027)$. The Fatigue-Inertia ratings did not differ for the placebo and no drink conditions $(p=0.415)$. The post-hoc LSD tests for the main effect of Time revealed that Fatigue-Inertia ratings increased with time, as ratings were significantly higher at 220, 280, and 340 minutes when compared to the first 40 minute measurement $(p<0.05)$.

Results of the ANOVA for the POMS Confusion-Bewilderment ratings revealed a significant main effect of Dose, $F(2,26)=5.55, p=0.010$, and a significant main effect of Time, $F(5,65)=4.17, p=0.002$. There was no significant interaction in this analysis $(p=0.33)$. Post-hoc LSD tests for the main effect of Dose revealed that Confusion-Bewilderment ratings were significantly higher following consumption of the energy shot when compared to the no drink condition $(p=0.007)$. There were no significant differences for the energy shot versus placebo condition $(p=0.095)$ and the placebo versus no drink condition ( $p=0.125)$. The post-hoc LSD tests for the main effect of Time revealed that Confusion-Bewilderment ratings decreased with time, as ratings were significantly lower at the 280 and 340 minute time points when compared to the first 40 minute measurement $(p<0.024)$.

Results of the ANOVA for the Tension-Anxiety ratings on the POMS revealed a significant Dose $\times$ Time interaction, $F(10,130)=1.95, p=0.044$. To understand the interaction further, separate repeated measures ANOVAs were conducted to examine the effect of Dose separately at each time point. There was a significant effect of Dose when examined at the first 40 minute time point, $F(2,26)=4.87, p=0.016$. Posthoc LSD tests for 40 minutes revealed that Tension-Anxiety ratings were significantly higher after the energy shot when compared to the no drink condition $(p=0.028)$. There was also a nonsignificant trend for Tension-Anxiety ratings to be higher after the energy shot when compared to the placebo condition $(p=0.057)$. The no drink and placebo conditions did not differ $(p=0.336)$. For the ANOVAs conducted to examine the effect of Dose at the other time points, there were no other significant effects $(p>0.38)$.

Finally, the results of the ANOVA for the POMS Depression-Dejection ratings revealed no significant main effects or interaction $(p>0.05)$. Similarly, the results of the ANOVA for the POMS Anger-Hostility ratings revealed no significant main effects or interaction $(p>0.13)$.

\section{Cued go/no-go task performance}

The mean RTs and p-inhibition failure scores for the cued go/no-go task were not significantly different at baseline $(p>0.12)$. Mean $(S D)$ RTs and p-inhibition failures scores following dose administration are presented in Table 3. Results of the ANOVA for mean RTs for the no-go cue condition revealed a significant main effect of Time, $F(5,65)=$ 
Table 3. Mean ( $S D$ ) Reaction Times (RTs) in the No-Go Cue Condition and P-INHibition FAILURES IN THE Go Cue Condition in the Cued Go No-Go Task for Each Time Point for the Energy Shot, Placebo, and No Drink Conditions

\begin{tabular}{|c|c|c|c|c|c|c|}
\hline \multirow[b]{3}{*}{ Variable } & \multicolumn{6}{|c|}{ Dose condition } \\
\hline & \multicolumn{2}{|c|}{ No drink } & \multicolumn{2}{|c|}{ Placebo } & \multicolumn{2}{|c|}{ Energy shot } \\
\hline & $M$ & SD & $M$ & SD & $M$ & SD \\
\hline \multicolumn{7}{|c|}{ Mean RTs (ms) } \\
\hline 30 minutes & 305.30 & 27.09 & 293.93 & 21.65 & 303.80 & 41.47 \\
\hline 0 minut & 315.21 & 35.21 & 307.13 & 36.43 & 307.63 & 46.60 \\
\hline 0 minutes & 314.93 & 43.10 & 304.14 & 43.74 & 313.10 & 38.30 \\
\hline & 315.93 & 40.52 & 312.87 & 42.09 & 318.68 & 49.55 \\
\hline ites & 321.72 & 45.39 & 310.54 & 35.38 & 302.61 & 42.09 \\
\hline 330 minutes & 318.66 & 38.02 & 308.63 & 37.32 & 316.64 & 65.27 \\
\hline \multicolumn{7}{|c|}{ Mean p-inhibition failures } \\
\hline 30 minutes & 0.10 & 0.08 & 0.06 & 0.09 & 0.06 & 0.06 \\
\hline & 0.1 & 0.17 & 0.13 & 0.11 & 0.09 & 0.10 \\
\hline & 0.1 & 0.18 & 0.11 & 0.11 & 0.13 & 0.12 \\
\hline & 0.16 & 0.1 & 0.11 & 0.13 & 0.15 & 0.13 \\
\hline & 0.13 & 0.18 & 0.15 & 0.19 & 0.15 & 0.14 \\
\hline 330 minutes & 0.15 & 0.20 & 0.16 & 0.15 & 0.17 & 0.12 \\
\hline \multicolumn{7}{|c|}{ Pulse rate (beats/minute) } \\
\hline & & & 68.93 & 11.48 & 70.14 & 10.95 \\
\hline 120 minutes & 65.64 & 11.86 & 67.00 & 11.16 & 68.43 & 11.32 \\
\hline & 63.79 & 10.8 & 66.21 & 11.64 & 67.43 & 10.52 \\
\hline 240 minut & 64.00 & 10.1 & 66.50 & 11.35 & 66.93 & 8.77 \\
\hline & 67.14 & 11.18 & 67.43 & 12.41 & 68.93 & 9.68 \\
\hline 360 minutes & 68.07 & 12.16 & 66.36 & 9.56 & 70.29 & 9.94 \\
\hline
\end{tabular}

Mean $(S D)$ pulse rate (beats/minute) readings for each time point for the energy shot, placebo, and no drink conditions.

$\mathrm{RT}$, reaction times.

3.49, $p=0.007$. There were no other significant effects for this analysis $(p>0.19)$. Post-hoc LSD tests examining the main effect of Time revealed that mean RTs were significantly slower at each time point, except 90 minutes, when compared to the first measurement at 30 minutes $(p<0.05)$.

Results of the ANOVA for p-inhibition failures for the go cue condition revealed a significant main effect of Time, $F(5,65)=5.20, p<0.001$. There were no other significant effects of this analysis $(p>0.52)$. Post-hoc LSD tests examining the main effect of Time revealed that $\mathrm{p}$-inhibition failures increased at each time point when compared to the first measurement at 30 minutes $(p<0.044)$.

\section{Blood pressure and pulse rate}

The mean systolic and diastolic blood pressure readings and pulse rate readings were not significantly different at baseline $(p>0.21)$, and the participants started sessions with a mean systolic/diastolic blood pressure reading of 116/71 mm Hg. Mean $(S D)$ blood pressure readings following dose administration are presented in Figures 1 and 2. Mean $(S D)$ pulse rate readings are presented in Table 3. Results of the ANOVA for systolic blood pressure readings revealed a significant main effect of Dose, $F(2,26)=13.66$, $p<0.001$, and a significant main effect of Time, $F(5$, $65)=4.42, p=0.002$. There was no significant interaction for this analysis $(p=0.69)$. Post-hoc LSD tests examining the main effect of Dose revealed that systolic blood pressure

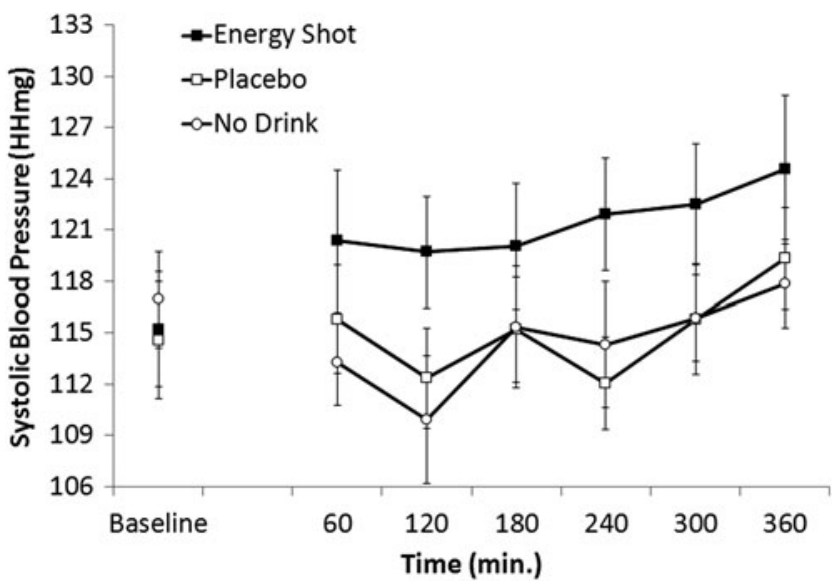

FIG. 1. Mean systolic blood pressure readings $(\mathrm{mm} \mathrm{Hg})$ at baseline and for each time point for the energy shot, placebo, and no drink conditions. Standard errors are represented in the figure by the error bars attached to each data point.

readings were significantly higher in the energy shot condition when compared to both the no drink and placebo drink conditions ( $p<0.003$; see Fig. 1). The placebo and no drink conditions did not differ $(p=0.61)$. Post-hoc LSD tests examining the main effect of Time revealed that systolic blood pressure readings were significantly higher at 360 minutes when compared to 60 minutes $(p=0.046)$.

Results of the ANOVA for diastolic blood pressure readings revealed a significant main effect of Dose, $F(2,26)=9.62$, $p<0.001$, and a significant main effect of Time, $F(5,65)=$ $2.74, p=0.026$. There was no significant interaction for this analysis ( $p=0.14$ ). Post-hoc LSD tests examining the main effect of Dose revealed that diastolic blood pressure readings were significantly higher in the energy shot condition when compared to both the no drink and placebo drink conditions $(p<0.003$; see Fig. 2). The placebo and no drink conditions did not differ $(p=0.51)$. Post-hoc LSD tests examining the main effect of Time revealed that diastolic blood pressure readings were significantly higher at 240 and 360 minutes when compared to 60 minutes $(p<0.02)$.

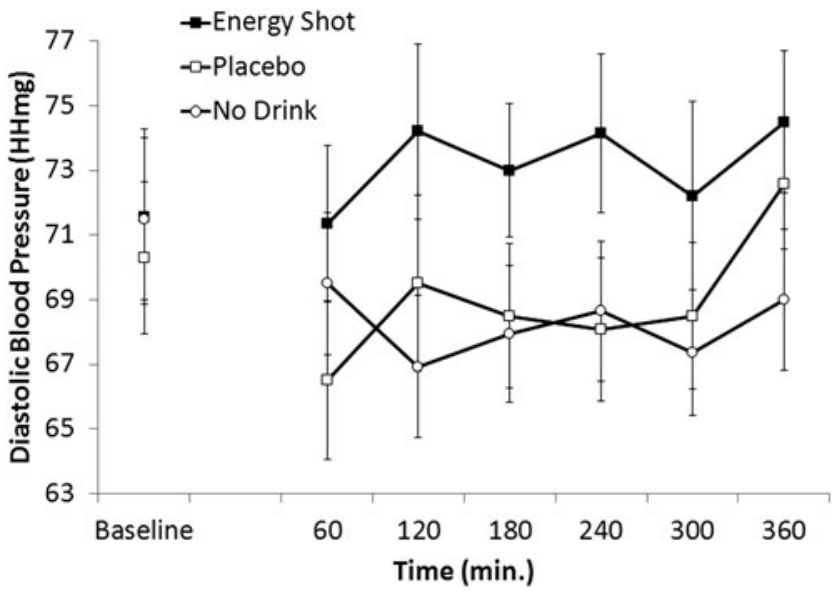

FIG. 2. Mean diastolic blood pressure readings ( $\mathrm{mm} \mathrm{Hg}$ ) at baseline and for each time point for the energy shot, placebo, and no drink conditions. Standard errors are represented in the figure by the error bars attached to each data point. 
Finally, results of the $3 \times 6$ ANOVA for pulse rate readings revealed a significant main effect of Time, $F(5,65)=5.18$, $p<0.001$ (see Table 3). There was no other main effect or interaction for this analysis $(p>0.16)$. Post-hoc LSD tests examining the main effect of Time revealed that mean pulse rate was significantly lower at 120, 180, and 240 minutes when compared to the 60 minute measurement $(p<0.03)$.

\section{Discussion}

This research examined the acute effects of an energy shot, when compared to both a placebo drink and a no drink control, on subjective state and objective performance on a behavioral control task. In addition, cardiovascular measures including blood pressure and pulse rate were recorded. The findings revealed that consumption of the energy shot did improve subjective state, as anticipated. Administration of the energy shot resulted in increased ratings of VigorActivity and decreased ratings of Fatigue-Inertia. The POMS ratings also revealed that the energy shot resulted in modest increased ratings of Tension-Anxiety early in testing. While subjective state was altered by the energy shot, it did not improve objective performance as measured by the cued go/no-go behavioral control task. Both mean RTs and p-inhibition failures worsened over time, regardless of whether the energy shot was administered. Finally, and most importantly, the energy shot elevated both systolic and diastolic blood pressure for a sustained period of many hours.

The results of this study suggest that consumers should weigh the relative benefits and costs when considering the use of an energy shot. While the consumer may perceive improvements in subjective state, the benefits may not extend to objective performance. Moreover, consumption of one energy shot in this study resulted in an elevation of systolic and diastolic blood pressure over a significant period of time. Patients with preexisting hypertension or other medical conditions, such as abnormal heart rhythm, where elevated blood pressure is contraindicated should be cautious about use of these new consumer products. In addition, energy products are popular with athletes who perceive that their use may enhance athletic performance. ${ }^{19,20}$ It is unknown whether energy shot related increases in blood pressure could potentially cause health problems for athletes. However, it remains to be seen if the findings of the current study, which used one energy shot, are replicated using different doses or different energy products. The manufacturer of the product used in the current study also has extra strength and decaffeinated versions of their product, suggesting that consumers could tailor their use to maximize benefits based on their needs and background characteristics. In addition, other research has demonstrated that energy drinks can facilitate some aspects of objective performance. For example, one study revealed that the lowest dose of an energy drink $\left(1.8 \mathrm{~mL} / \mathrm{kg}\right.$ or half a $250 \mathrm{~mL}$ can of Red Bull $\left.{ }^{\circledR}\right)$ resulted in the greatest improvements in reaction times on the same cued go/no-go task used in the current study. ${ }^{1}$ While these two energy products were not directly compared in one study, it would be interesting for future research to examine if less is more when it comes to the facilitating benefits of doses of caffeine.

In sum, this study adds to a growing literature that suggests that the benefits of energy product use may be somewhat limited to improvements in subjective state and thus may not outweigh the potential health risks, particularly for vulnerable individuals. ${ }^{21-30}$

\section{Acknowledgments}

This research was supported by National Institutes of Health (NIH) grants AA019795 and GM103436 awarded to CA Marczinski. The funding sources had no other involvement in this research other than financial support. The content is solely the responsibility of the authors and does not necessarily represent the official view of National Institute of Alcohol Abuse and Alcoholism, the National Institute of General Medical Sciences, or the National Institutes of Health.

\section{Author Disclosure Statement}

No competing financial interests exist.

\section{References}

1. Howard M, Marczinski C. Acute effects of a glucose energy drink on behavioral control. Exp Clin Psychopharm 2010; 18:553-561.

2. Eudy A, Gordon L, Hockaday B, et al. Efficacy and safety of ingredients found in preworkout supplements. Am J Health Syst Pharm 2013;70:577-588.

3. Barone J, Roberts H. Caffeine consumption. Food Chem Toxicol 1996;34:119-129.

4. Substance Abuse and Mental Health Services Administration, Center for Behavioral Health Statistics and Quality. The DAWN report: update on emergency department visits involving energy drinks: a continuing public health concern. 2013. Available at www.samhsa.gov/data/2k13/DAWN126/ sr126-energy-drinks-use.htm. Accessed January 20, 2014.

5. Seifert S, Seifert S, Schaechter J, et al. An analysis of energy-drink toxicity in the National Poison Data System. Clin Toxicol 2013;51:566-574.

6. Scholey A, Kennedy D. Cognitive and physiological effects of an "energy drink": an evaluation of the whole drink and of glucose, caffeine and herbal flavouring fractions. J Psychopharmacol 2004;176:32-330.

7. Snel J, Lorist M. Effects of caffeine on sleep and cognition. Prog Brain Res 2011;190:105-117.

8. Childs E, de Wit H. Enhanced mood and psychomotor performance by a caffeine-containing energy capsule in fatigued individuals. Exp Clin Psychopharm 2008;16:13-21.

9. Einother S, Giesbrecht T. Caffeine as an attention enhancer: reviewing existing assumptions. J Psychopharmacol 2013; 225:251-274.

10. Kennedy D, Scholey A. A glucose-caffeine 'energy drink' ameliorates subjective and performance deficits during prolonged cognitive demand. Appetite 2004;42:331-333.

11. Nehlig A. Is caffeine a cognitive enhancer? J Alzheimers Dis 2010;20:S85-S94.

12. Marczinski C, Fillmore M. Dissociative antagonistic effects of caffeine on alcohol-induced impairment of behavioral control. Exp Clin Psychopharm 2003;11:228-236.

13. Higgins J, Babu K. Caffeine reduces myocardial blood flow during exercise. Am J Med 2013;126:730.e1-8.

14. Mesas A, Leon-Munoz L, Rodriguez-Artalejo F, LopezGarcia E. The effect of coffee on blood pressure and cardiovascular disease in hypertensive individuals: a systematic review and meta-analysis. Am J Clin Nutr 2011;94:11131126. 
15. Seifert S, Schaechter J, Hershorin E, Lipshultz S. Health effects of energy drinks on children, adolescents, and young adults. Pediatrics 2011;127:511-528.

16. McNair D, Lorr J, Dropplemann L. Profile of Mood States Brief Form. North Tonawanda, NY: Multi-Health Systems, Inc., 2003.

17. McNair D, Heuchert J. Profile of Mood States: Technical Update. North Tonawanda, NY: Multi-Health Systems, Inc., 2005.

18. Schneider W, Eschman A, Zuccolotto A. E-Prime User's Guide. Pittsburgh, PA: Psychology Software Tools, 2002.

19. Miller K. Wired: energy drinks, jock identity, masculine norms, and risk taking. J Am Coll Health 2008;56:481-490.

20. Hoyte C, Albert D, Heard K. The use of energy drinks, dietary supplements, and prescription medications by United States college students to enhance athletic performance. J Commun Health 2013;38:575-580.

21. James J, Gregg M. Effects of dietary caffeine on mood when rested and sleep restricted. Hum Psychopharmacol Clin Exp 2004;19:333-341.

22. Arria A, Caldeira K, Kasperski S, et al. Energy drink consumption and increased risk for alcohol dependence. Alcohol Clin Exp 2011;35:365-375.

23. Arria A, O'Brien M. The "high" risk of energy drinks. JAMA 2011;305:600-601.

24. Avci S, Sarikaya R, Buyukcam F. Death of a young man after overuse of energy drink. Am J Emerg Med 2013; $31: 1624$.
25. Berger A, Alford K. Cardiac arrest in a young man following excess consumption of caffeinated "energy drinks." Med J Aust 2009;190:41-43.

26. Goldfarb M, Tellier C, Thanassoulis G. Review of published cases of adverse cardiovascular events after ingestion of energy drinks. Am J Cardiol 2014;113:168-172.

27. Rottlaender D, Motloch L, Reda S, Larbig R, Hoppe U. Cardiac arrest due to long QT syndrome associated with excessive consumption of energy drinks. Int J Cardiol 2012; 158:e51-e52.

28. Sepkowitz K. Energy drinks and caffeine-related adverse events JAMA 2013;309:243-244.

29. Temple J. Caffeine use in children: what we know, what we have left to learn, and why we should worry. Neurosci Biobehav Rev 2009;33:793-806.

30. Terry-McElrath Y, O’Malley P, Johnston L. Energy drinks, soft drinks, and substance use among United States secondary school students. JAMA 2014;8:6-13.

Address correspondence to:

Cecile A. Marczinski, PhD

Department of Psychological Science Northern Kentucky University 349 MEP, 1 Nunn Drive Highland Heights, KY 41099

E-mail: marczinskc1@nku.edu 
